\title{
Where does that [EyeGaze] go?
}

\author{
Elena Benedicto (Purdue University) \\ ebenedi@purdue.edu \\ Paula J. Rodriguez-M (Universidad Nacional de Colombia) \\ pajrodriguezmo@unal.edu.co \\ Esther Rivera (Purdue University) \\ rivera82@purdue.edu
}

\begin{abstract}
In this paper we analyze the non-manual marker EyeGaze as a grammatical function marker in motion predicates in American Sign Language (ASL). We detect the use of EyeGaze in endpoint locatives only (as opposed to potential goal locatives) and in the head of the classifier predicate itself. Along the lines of Nichols' (1986) typology, we identify the former as GF-marking on the constituent (that is, Case) and the latter as GF-marking on the Head (that is, agreement). We also identify the coexistence of these two patterns in the same utterance: Case in the endpoint locative and agreement on the classifier. Therefore, ASL falls under the Double-Marking language type. In this respect, thus, ASL aligns with crosslinguistic patterns documented elsewhere.
\end{abstract}

Keywords: non-manual markers, EyeGaze, motion predicates, Case, agreement

\section{Introduction}

In this paper we address the use of the non-manual marker eye gaze (henceforth, NMMEyeGaze or EyeGaze) as a grammatical function marker (GF) in utterances involving motion predicates. The goal is twofold: first, to identify, systematize and account for any new distributional patterns concerning the NMM-EyeGaze in our motion predicate dataset for American Sign Language (ASL); second, to provide a (more) comprehensive, accurate and coherent analysis of the range of morphosyntactic uses of NMM-EyeGaze. Ultimately, we aim to undertake these two goals within the framework of crosslinguistic and cross-modality variation and to evaluate how this phenomenon situates sign languages within the range of variation observed in natural Human Language.

In this work, thus, we are interested in the uses of EyeGaze that are grammatical in nature, and in particular those that can be identified as functional (or inflectional) in nature (thus, not affective uses of EyeGaze); in particular, we are interested in investigating EyeGaze as a marker of grammatical function(s). For that, we will first do an overview of the typological ways in which natural languages manifest the morphology of grammatical functions (Section 2.1), and how EyeGaze has been understood in the sign language literature (Section 2.2). We tie this background into a hypothesis in Section 3 which leads into the discussion of our results in Section 5, after Section 4 presents the data collection methods. Section 6 wraps 
up the discussion, evaluates the hypothesis in Section 3 and cycles it back to the place of EyeGaze as a marker of grammatical functions within the whole range of variation manifested in natural languages.

\section{Background on EyeGaze and morphological marking of gram- matical functions}

In this section we provide the basic background to understand the mechanisms for GFmarking and previous accounts of EyeGaze in sign languages.

\subsection{On grammatical marking variation cross-linguistically}

In her paper based on a substantial sample of languages across the world, Nichols (1986) identifies a 3-way distinction in the way that languages use morphology to mark grammatical functions. In her study, she distinguishes languages that:

(1) (a) morphologically mark GF on the head of the unit (the verb-predicate in a sentence, for instance);

(b) use a GF-morpheme on the dependent (i.e., the associated constituent itself) rather than on the head; and

(c) mark both the head and the dependent (i.e., the constituent) with some sort of GF-morpheme each.

The a-type identifies head-marking languages; the second type, dependent-marking languages and the third type are double-marking languages. In other words, and using more common terms for predicates, head-marking would be understood as agreement morphemes on the verb; dependent-marking would be understood as Case morphemes on the associated constituent; and double-marking would render both a Case morpheme on the constituent and an agreement morpheme on the verb. In this paper we will, for clarity, use the term constituent-marking for the second type. Here are some examples:

(2) a-xàc'a a-phò̀s a-š $\mathrm{q}^{\circ}$ ̀̀ $\quad$-lò-y-te-yt'.

the-man the-woman the-book it-to.her-he-gave-FINITE

'The man gave the woman a book.' Abkhaz (Nichols 1986, 73a)

(3) $\mathrm{Cu}$ stag-a zudčun-na kni:ga-ø j-elira.

DEM.OBL person-ERG woman-DAT book-Nom $j$-gave

'The man gave the woman a book.' ${ }^{1}$ Chechen (Nichols 1986, 73b)

(4) Emakume gazte-a-k ume txiki-a-ri liburu zahar bat.ø eman

Woman young-DSG-ERg child small-DSG-DAT book old one.ABs give

dio.

Aux.3SDAT.3SABS.3SERG

'The young woman gave an old book to the small child.' Euskera [Batua] (Ager

Gondra, pc)

1. Johanna Nichols (p.c.) explains that, while nouns in Chechen have eight Cases, their determiners and other modifiers only have two, nominative and 'all-purpose' oblique. That's why the D $c u$ is glossed as OBL despite modifying the ERG staga. 
The a-type, head-marking languages, is exemplified in (2) with an Abkhaz sentence: we can see that the verb is the one having morphological affixes indicating the GF of the constituents

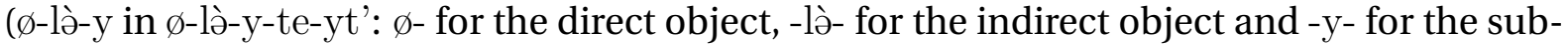
ject). The verb in Abkhaz, thus, agrees with the object, indirect object and subject; the constituents themselves do not carry any markings. Variations within this group may include how many arguments are marked on the head/verb and what kind of morpheme is used (affix/clitic/...). Other languages included in this group are French, Spanish and Italian.

The b-type, on the other hand, a constituent-marking language, is exemplified in (3) with a Chechen utterance: contrary to (2), in Chechen, the constituents are the ones carrying GFmorphemes (e.g., - $a$ for the Ergative, -na for the Dative and - $ø$ for the Nominative) but the verb $j$-elira does not include any particular morpheme related to those constituents. This is what we usually interpret as a Case-marking language. Variations in this group may include whether the Case marking is in the form of a phrasal particle (as in Korean or Japanese) or in the form of morphemes attached to all elements in the constituents (as in Russian or Latin).

The c-type, finally, double-marking languages, is illustrated in (4), with an example from Euskera-Batua. In (2) we can observe GF morphemes on both the constituents (i.e., the Case markers $-k$ for the Ergative, - $r i$ for the Dative, $-\varnothing$ for the Absolutive) and the head (the portmanteau agreement morpheme for $3^{\text {rd }}$ person singular Dative, $3^{\text {rd }}$ person singular Absolutive and $3^{\text {rd }}$ person singular Ergative, 3SDAT.3SABS.3SERG, appearing in the Auxiliary dio). Another example of this kind of double-marking language is Yup'ik (Jacobson 1995).

This three-way crosslinguistic distinction will be pertinent in our discussion about EyeGaze in ASL as we ascertain whether its GF use corresponds to a constituent marking (that is, Case) or to head marking (that is, agreement), or both (double-marking). Let us first examine previous work about EyeGaze as a grammatical marker in sign languages.

\subsection{On the interpretation of EyeGaze as grammatical marking}

Neidle et al. (2000), and Bahan (1996) before, identified EyeGaze on a constituent as an agreement marker in all three types of verbs in ASL (plain, agreeing and spatial). Later on, Thompson, Emmorey, and Kluender (2006), based on experimental data, found EyeGaze as agreement only in conjunction with manual agreement (thus, not plain verbs) and for both objects and for locatives in spatial verbs (though with no specification on the type of locative).

Hosemann (2013) for DGS confirms the pattern but finds it to be not as systematic as was found for ASL. And Calderón Verde et al. (2018), working on LSCu (Sign Language of Cuba), found EyeGaze in motion predicates both on the internal argument and on the endpoint locative in telic predicates.

This brief overview paints a fine-grain syntactically-driven variation that is reminiscent of works like Quer (2010) and Quadros and Quer (2011) or Lourenço (2018), that address the variability of agreement markers in sign languages as part of normal variation in the patterns of natural languages.

\section{Hypothesis}

For this paper, we are going to evaluate the hypothesis that EyeGaze in ASL is a GF morphological marker. If such a GF-marker appears on a constituent, then, according to the typology of Nichols (1986), it can be considered constituent-marking and thus Case (i.e., (1b) and (3)). If, on the other hand, EyeGaze, as a GF-marker, appears on the verb/predicate, then it can be considered head-marking and thus agreement (i.e., (1a) and (2)). This is summarized in (5): 
EyeGaze as a grammatical function marker

1. if $\mathrm{EG}$ on the constituent $\rightarrow \mathrm{EG}$ is Case

$[(1 b) /(3)]$

2. if $\mathrm{EG}$ on the predicate $\rightarrow \mathrm{EG}$ is agreement

$[(1 \mathrm{a}) /(2)]$

For our analysis, we are going to assume Benedicto and Brentari's (2004) analysis of classifiers, as well as the more specific analysis in Benedicto (2018), and the works by Calderón Verde et al. (2018) on EyeGaze in LSCu (Sign Language of Cuba). In particular, and as relevant for this work, we are going to assume that in classifier predicates, the movement component corresponds to the eventive element of the utterance, with the handshape as a functional morpheme, related to but distinct from, the actual nominal constituent or argument. We also assume a subeventive decomposition of predicates (along the lines of Ramchand 2008 and Borer 2005); and, in particular, for motion predicates, we assume a subeventive decomposition realized as a Serial Verb Construction (with a process subevent and a telic subevent), as in Benedicto, Branchini, and Mantovan (2015).

\section{Methods}

Data were collected from 3 adult native signers of ASL who were recorded by a Deaf ASL signer, using a self-paced video-clip application (Benedicto 2019) designed for crosslinguistic and cross-modality language elicitation of motion predicates. The application is constituted by 175 animated video-clips, sorted around 19 different series (about a bird, a goose, a child, a paper plane, etc.; i.e., \pm animate/ \pm human), randomly divided into 7 different blocks. The video-clips include minimally contrastive pairs encoding variables related to motion predicates: 3D path-trajectory, telicity, result state and agentivity. The application is designed to facilitate data elicitation for a qualitative analysis, not experimental in nature, and was initially created as part of a larger crosslinguistic/crossmodality study, including sign and spoken languages from different typological groups. The goal of the application is to discover what the grammar of any given language can produce and under which conditions, not preference or frequency; that is, it is a tool for grammatical description.

Each signer was asked to say what happened in the video-clip, and were not limited in the number of responses they chose to provide. The recordings were done from two perspectives, one with a frontal view and another at a $45^{\circ}$ side angle. The two recordings were synchronized and clipped, and each clip was processed in ELAN using a tier-template designed as part of the larger cross-linguistic and cross-modality project on motion predicates.

\section{Results and analysis}

In this section we report on two patterns of EyeGaze identified in the dataset: (a) EyeGaze on the locative constituent and (b) Eyegaze on the predicate itself (the eventive component of the utterance). The existence of these two patterns, alongside the hypothesis in (5) in Section 3, would indicate that ASL falls under the double-marking language type within the crosslinguistic typology set by Nichols (1986) (i.e., (1c)/(4)): languages that mark both the constituent and the head of the syntactic unit (the verb/predicate in this case).

Section 5.1 reports on the first pattern (EyeGaze on the locative constituent) and Section 5.2 reports on the latter (EyeGaze on the predicate). 


\subsection{EG on the locative constituent: Case}

This pattern found in the ASL dataset identifies EyeGaze marking on the endpoint locative, and given that it is marking on the constituent, identifies it as an instantiation of constituentmarking; thus, following the hypothesis in (5), as an instantiation of Case.

The example and screenshots in (6) provide details for this EyeGaze marker type. ${ }^{2}$ The light gray line marks the EyeGaze and the broken purple line indicates the trajectory of the movement. At the end of example (6) (the initial grey transcription indicates the previous context), we see the signer's EyeGaze (gray line) directed at the endpoint locative in the nondominant hand H2 ('1:5].i+BE_AT. $\gamma$ lc' for SMALL-TREE) while the figure is moving in the space tracing the trajectory (indicated by the broken purple line; a. and b. screenshots); finally, upon the figure's reaching of the endpoint, the signer closes her eyes and looks away to rest back at the computer (c. screenshot).

(6) EyeGaze on endpoint locative [0112_ASLSN]

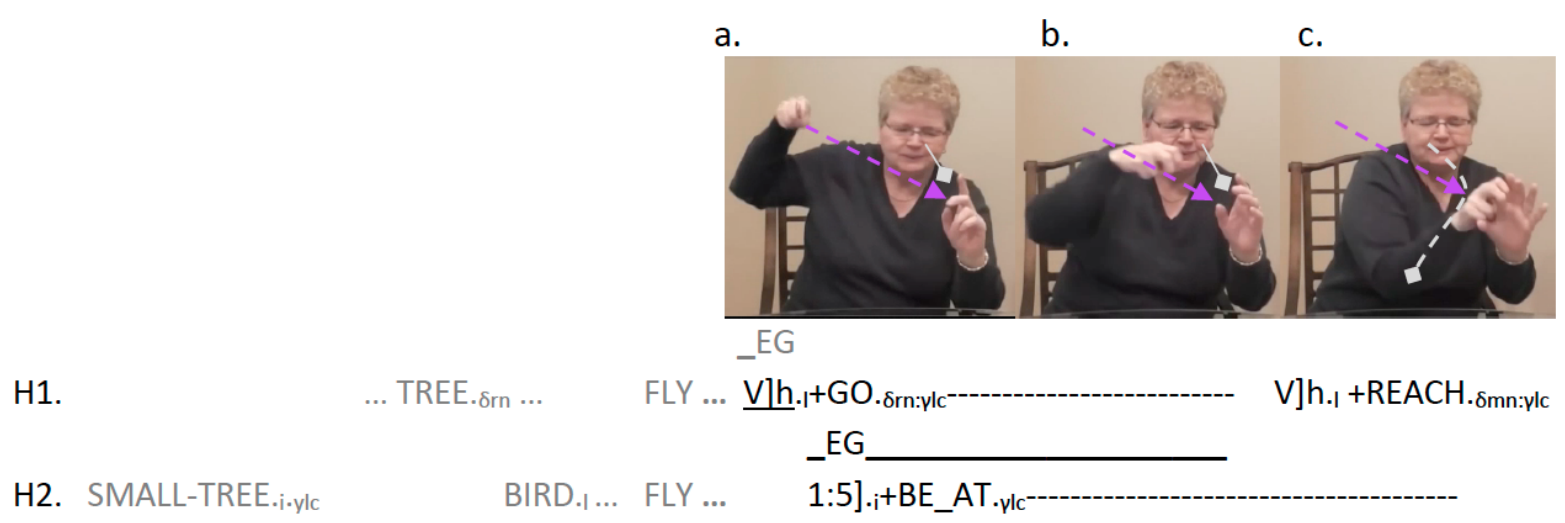

'There is a small tree (to the left); a bird goes flying down all the way to the small tree.'

Along the lines of Rodríguez-Monroy's work (2021, adopting previous work carried out by Calderón Verde et al. 2018 for LSCu), the EyeGaze directed at the locative is a morphological marker present only in telic predicates and not in atelic ones. In Benedicto, Branchini, and Mantovan (2015) for LIS, it was proposed that telicity arises out of a complex Serial Verb Construction including a syntactic subcomponent with a REACH head (V]h.1+REACH. $\delta m n: \gamma l c$ in (6c)). The locative appears as a syntactic sister to that REACH head, receives the EyeGaze marking, leading to the interpretation of the locative as an endpoint and of the predicate as telic - as is the case in (6) above.

This approach predicts that in atelic predicates, there will be absence of the subeventive head REACH. Any potential locative will thus not receive EyeGaze and the resulting interpretation will be that of a potential goal ('towards') yielding an atelic reading for the predicate. This is exactly what we can observe and confirm in (7) below:

2. Examples are transcribed using one line for H1 (the dominant hand) and another for H2 (the nondominant hand), time-aligned. In particular, for classifiers, the portion of the transcription preceding "+" refers to the handshape (e.g., V]h), with the subindex indicating co-referentiality with the constituent (.j), the transcription following “+” refers to the eventive element (+GO, +BE_AT) followed by a space subindex $(. \delta r n: \gamma l c, \gamma l c)$ that follows the system in Benedicto, Branchini, and Mantovan (2015). Space subindices include specification for three space planes (vertical: $\delta$-high, $\gamma$-neutral, $\beta$-low; horizontal: l-left, m-medial, r-right; deictic: n-near, c-central, f-far); two space subindices separated by a “:” indicate space location 'from' and space location 'to.' 
(7) No EyeGaze on locative in atelic predicate [0103_ASLEM]

a.

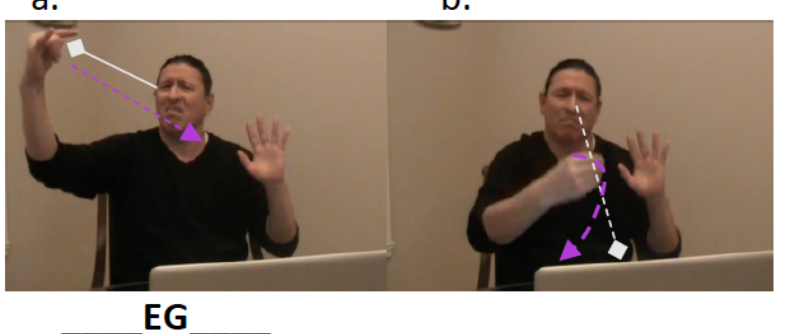

EG

H1. BIRD.j... FENCE.k•pln V]h.j+TAKE-OFF.8rf

H2. FENCE 5.k+BE_AT. $ү$ In
DISAPPEAR

'(A bird ... goes flying toward the fence.'

In (7) (again, with grey indicating previous context to screenshots in a. and b.), EyeGaze is never directed at the locative articulated by the non-dominant hand $\mathrm{H} 2\left(5 \cdot_{k}+\mathrm{BE}_{-} \mathrm{AT} \cdot \gamma l n\right)$. EyeGaze is first, ( 7 a), directed towards the trajectory predicate (L-I. $j+$ GO. $\delta r f: \gamma m n$ ) articulated in $\mathrm{H} 1$ (see next section for a discussion of this type of case) and then before the trajectory reaches the locative in $\mathrm{H} 2$ ( $7 \mathrm{~b}$ ), $\mathrm{H} 1$ articulates the sign DISAPPEAR (indicating the fading away of the image) and the EyeGaze goes to rest back at the computer. Without the syntactic subeventive head REACH, and without EyeGaze on the locative, the predicate can only be interpreted as atelic, as predicted.

This contrast between the two types of locative, the endpoint locative in telic predicates marked by EyeGaze and the potential-goal locative in atelic predicates not marked by EyeGaze, constitutes a fine-grain distinction over the results of Thompson, Emmorey, and Kluender (2006), who did not consider the contribution of Aktionsart in the interpretation of the locative constituent, and confirm results reported in Calderón Verde et al. (2018) for LSCu.

\subsection{EG on the predicate: a pattern of agreement}

A new pattern, crucial for hypothesis (5), following Nichol's (1986) typology, is also identified in our dataset: it finds EyeGaze on the movement of the motion predicate. This pattern has not been previously identified and it constitutes marking on the eventive component of the classifier predicate, that is, the head of the utterance. As such (that is, as an instantiation of head-marking), and according to the hypothesis in (5) it would constitute a case of agreement. The data in our dataset contain three different subtypes of this pattern, as summarized in (8):

(8) EyeGaze on the Predicate head. Types:

a. EyeGaze on the hand, as it articulates the movement of the predicate;

b. EyeGaze on the space between the hands, as they articulate the movement of the predicate;

c. EyeGaze on the (unrealized) projection of the trajectory, to be later articulated by the movement of the hands.

\subsubsection{Subtype (8) [a]: EyeGaze on the hand}

On the first subtype, (8) [a.], EyeGaze lies on the hand while articulating the movement of the classifier. This holds for both stative predicates (of the type BE_AT) as in (9), subtype [a]-i., 
and for eventive predicates, subtype [a]-ii, as in (10) of the type MOVE/GO/COME, denoting the trajectory or path, the process subevent, or as in (11) of the type REACH, denoting the telic subevent of a motion predicate. The screenshots depict the predicate of the utterance; as in previous examples, transcriptions in grey mark surrounding discourse, the light gray line marks the EyeGaze and the broken purple line indicates the trajectory of the movement:

(9) Subtype [a]-i. EyeGaze on Hand-in-Movement: Stative predicate [0102_ASLSN]

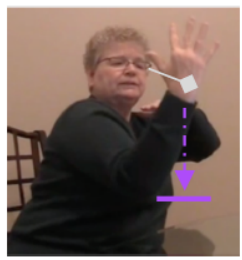

H1. IX.prf ... TREE.j ... $\overline{\text { 5.j+BE__AT.prf }}$

'(A tree) is located [there, far on the right].'

In (9) we see the signer laying her EyeGaze on H1 while this hand is articulating a short downward movement. This short, sharp downward movement has been identified (Benedicto and Brentari 2004, adopting previous work by Brentari 1993) as a kind of epenthetic movement associated with existential/locative predicates of the type BE_AT. ${ }^{3}$ In our dataset, this type of existential/locative predicate is pervasive while setting the (syntactic) participants in the signing space. Both TREE and the location (indicated by IX. $\gamma r f$, far right) had been previously identified in the utterance discourse.

The following example instantiates a case of EyeGaze on the movement, for an eventive predicate, encoding the trajectory: ${ }^{4}$

(10) Subtype [a]-ii. EyeGaze on Hand-in-Movement: Eventive predicate [0105_ASLEM]

a.

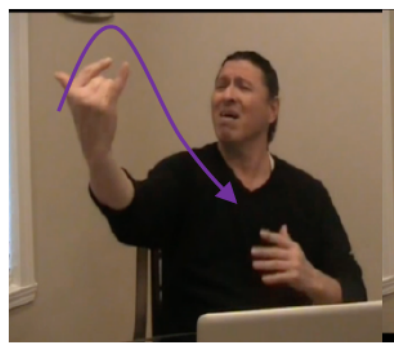

b.

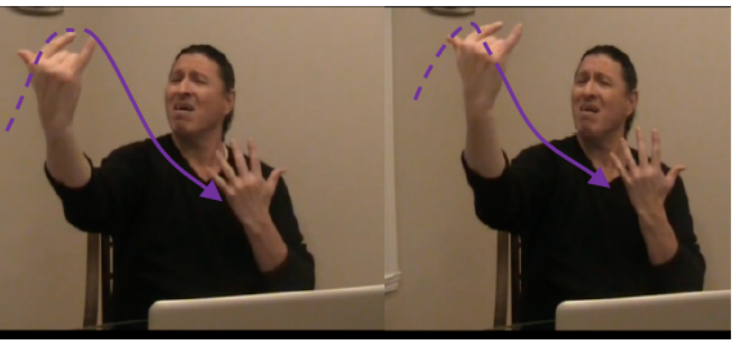

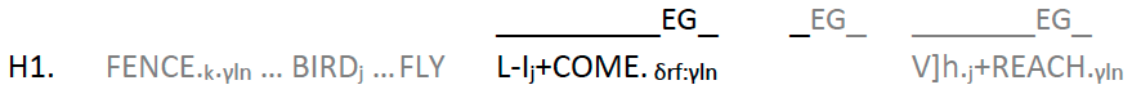

H2. FENCE

FLY

$5 . k+B E \_A T \cdot y l n--$

'(A bird) is coming (all the way up) to the fence.'

In this second case [a]-ii., the signer's EyeGaze lays on $\mathrm{H} 1$ while this hand is engaged in articulating the movement corresponding to the trajectory (indicated by the purple line)

3. For earlier work establishing that these are in fact stative predicates, see Supalla's (1982) dissertation.

4. Here we use a combination of broken and solid purple line to indicate the movement already done (broken) and the movement coming up (solid), to facilitate the visualization of the movement. (10a-c) correspond to the transcription in black. 
of the motion predicate (L- $\left.{ }_{j}+\mathrm{COME} \cdot \delta r f: \gamma l n\right)$. The EyeGaze follows $\mathrm{H} 1$ while it displaces in space, as the progression in screenshots a., b. and c. is intended to portray.

The trajectory ends on the endpoint in $\mathrm{H} 2$, as the $\mathrm{H} 1$ articulates the REACH head of the telic subcomponent. In fact, before $\mathrm{H} 1$ reaches $\mathrm{H} 2$, EyeGaze, which was following the trajectory of the movement as in (10), is re-directed to $\mathrm{H} 2$ articulating the endpoint locative, as in (11a), and then follows the articulation of the REACH head, as can be observed in (11b).

[0105_ASLEM]

a.

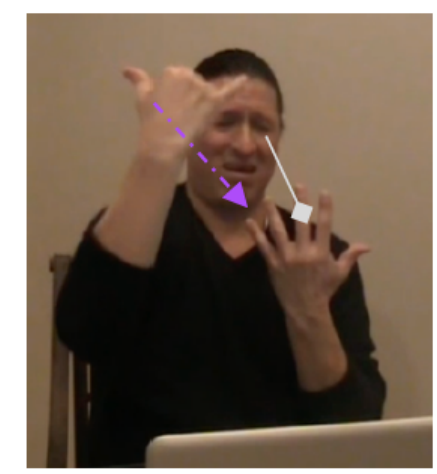

EG__ _ _EG_ b.

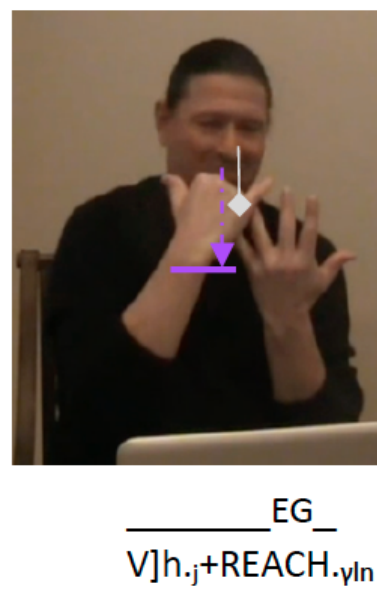

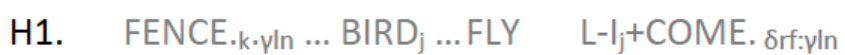

H2. FENCE

'(A bird) is coming (all the way up) to the fence.'

This last step exemplifies another instance of (8) [a]-ii (EyeGaze on the hand, as it articulates the movement of the predicate). Whereas EyeGaze in (10) focuses on the path or trajectory, the process subevent of a motion predicate, in (11) EyeGaze lands on the telic subeventive REACH head as it is articulating the telic subcomponent, as can be observed in (11b). In her work, Rodriguez-Monroy (2021) identifies three subtypes of EyeGaze associated with telicity: (1) EyeGaze on the endpoint locative only (Section 5.1, earlier); (2) EyeGaze on the REACH head only; and (3) EyeGaze on both the endpoint locative and the REACH head. ${ }^{5}$ Rodriguez-Monroy's (2) and (3) subcases would be instances of this subpattern (8) [a] (EyeGaze on the hand, as it articulates the movement of the predicate).

And in fact, Rodriguez-Monroy's subcase (3), as in (11a-b), would exemplify a case of double-marking since both the endpoint locative (in (11a)) and the head REACH (in (11b)) would be marked by EyeGaze.

While the three previous examples of the first subtype, (8) [a], cannot in fact elucidate whether the EyeGaze is directed at the hand or at the movement (the eventive component proper) since they are simultaneously co-articulated, the following two subtypes, (8) [b] and (8) [c], are more enlightening, clearly distinguishing between hands and movement/trajectory and pointing to the movement as the target of the EyeGaze.

\subsubsection{Subtype (8) [b]: EyeGaze on the space between the hands}

The second subpattern, (8) [b], has EyeGaze following the space in between $\mathrm{H} 1$ and $\mathrm{H} 2$ as they articulate the movement of this extension classifier predicate:

5. Subcase (3) seems to be the more prolific one, with $67.74 \%$ of the cases analyzed in Rodriguez-Monroy (2021). Subcase (1) with EyeGaze on the REACH head only, constitutes $6.45 \%$ and subcase (2), with EyeGaze only on the endpoint locative, $25.81 \%$. 


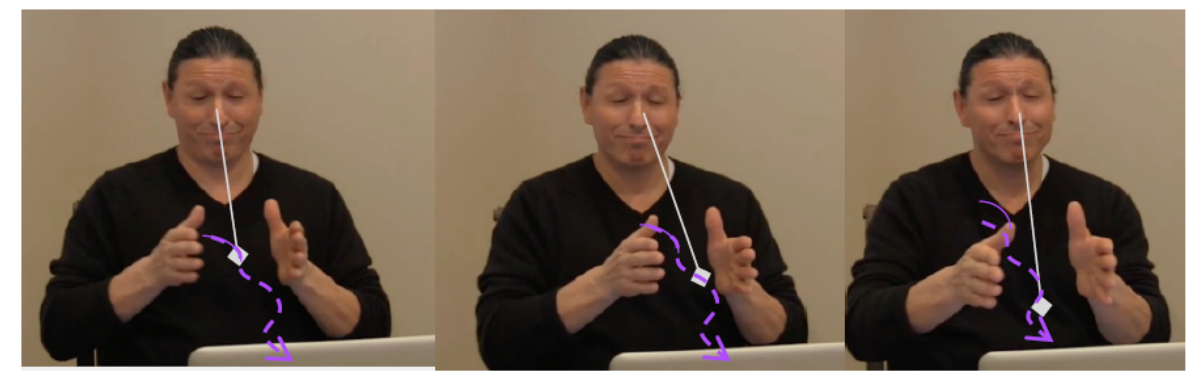

$$
\begin{aligned}
& \text { H1. RIVERj ... }
\end{aligned}
$$

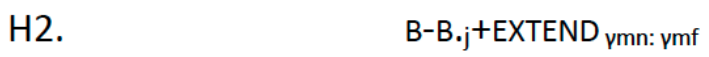

'(The river) extends in a winding way.'

In (12), the signer's EyeGaze is clearly not laying on the articulating hands, but on the space delimited by them, and crucially it follows the movement that denotes the extension of the river course. While the hands may be argued to denote the banks or the edge of the river, it is clear that the EyeGaze is not focused on them but on the river course trajectory being denoted by the space in between them. In this case, as opposed to the previous two, one can more clearly ascertain the difference between the hands, $\mathrm{H} 1$ and $\mathrm{H} 2$, and the movement components of the classifier predicate. It is clearer, then, that EyeGaze is directed towards the movement component, that is, the eventive component of this morphologically complex predicate and, therefore, its head.

This subpattern, unsurprisingly, happens with 2-handed predicates (since the two hands define and delimit the relevant space), mostly with extension classifiers (e.g., those used for RIVER and FENCE).

\subsubsection{Subtype (8) [c]: EyeGaze on the (unrealized) projection of the trajectory}

The third subpattern, (8) [c], finds EyeGaze landing on successive projections of the trajectory, before the hand actually traces the trajectory. This is the clearer subtype supporting the claim that EyeGaze is associated with the movement of the predicate, that is, its head. And, as such, it constitutes head-marking, and as head-marking and via hypothesis in (5), agreement. The utterance in (13) is an example of this third subtype [c]:

(13) Subtype [c]. EyeGaze on Projection-of-Movement: Trajectory [0111_ASLEM]

H1.

H2. TREE.I A.|+BE_AT.plc -------

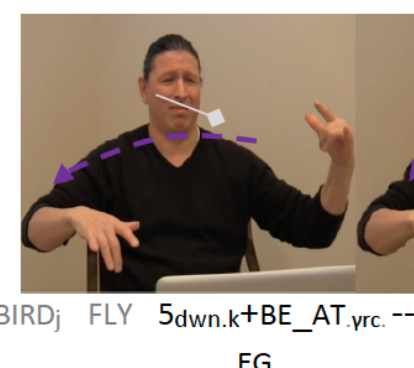

EG

FLY L-I.j+GO.plc:prc[-3]

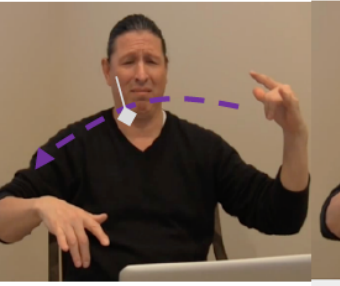

EG

plc:prc [-2]

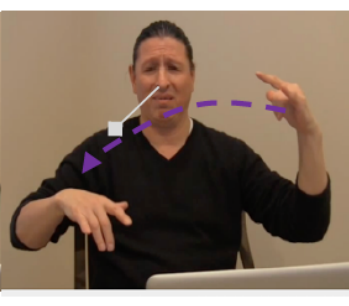

EG_-

'A bird is coming from a tree on the left to a tree on the right.' 
In (13) EyeGaze begins close to the articulation of $\mathrm{H} 2$ as it is beginning to engage in the movement that will delineate the trajectory of the predicate (indicated by $\gamma l c: \gamma r c[-3]$, in the transcription, with $\gamma l c: \gamma r c$ the intended trajectory (from origin $\gamma_{l c}$ to destination $: \gamma r c$, and ${ }_{[-3]}$ suggesting 3 degrees before destination). EyeGaze then jumps ahead and follows the, as of yet, unrealized trajectory of the movement as $\mathrm{H} 2$ follows behind (indicated in the transcription by, subsequently, $[-2]$ and $\left.{ }_{[-1]}\right)$.

Clearly, in this case, EyeGaze is not focused on the hand(s) but on the movement depicting the trajectory; more precisely, on the potentiality of that trajectory, the projection of the trajectory initiated by the $\mathrm{H} 2$ but not yet actualized. This subpattern occurs both with telic and atelic predicates, the latter both with a potential goal and without a potential goal.

The patterns uncovered and analyzed in this Section 5.2 systematize the function of EyeGaze on the classifier itself, thus clarifying statements in, e.g., Emmorey, Thompson, and Colvin (2008) about EyeGaze on the hands and EyeGaze on classifiers. The patterns we identify here are systematic across signers and across stimuli; that is, each individual signer presents samples of all of them and individual prompts can be responded to with all three patterns [a-c] in (8). We have not, as of yet, identified any particular pattern that requires, say, [a] over [c] or vice versa, but it is a matter that deserves further exploring.

The patterns of EyeGaze on the movement identified in this Section 5.2. are here interpreted as an instance of head-marking and, thus, agreement.

Looking now at the whole pattern of EyeGaze identified in Sections 5.1 and 5.2, we can state that ASL manifests patterns of (i) constituent-marking (in the endpoint locatives in Section 5.1), (ii) head-marking (in the patterns in Section 5.2), and (iii) double-marking in those cases where both the endpoint locative and the REACH head receive EyeGaze (as in example (11)).

\section{Conclusions}

In this paper we have examined the role of EyeGaze as a morphological marker in motion predicates; in particular we have evaluated its role as a marker of grammatical function (GF). In that sense and following both the early crosslinguistic typological work of Nichols (1986) and previous work on the role of EyeGaze in sign languages, we have looked at the contrast between EyeGaze directed at the articulation of the predicate head itself and EyeGaze directed at the articulation of an argument (in particular the endpoint locative argument of the motion predicate). We formalized these ideas in the hypothesis in (5): when directed at the head of the predicate itself, EyeGaze constitutes agreement; when directed at the (locative) argument, a constituent, it represents Case (again, following commonly accepted generalizations about GF-marking as developed in Nichols 1986).

What we have observed in our data is a variety of subpatterns for the first case (EyeGaze directed at the head of the predicate), most interestingly showing that EyeGaze can go on the trajectory of the predicate itself and not necessarily on the hands - thus confirming that EyeGaze is marking the eventive part of the predicate head (as per Benedicto and Brentari's 2004 analysis of classifiers). For the second case, we have corroborated a pattern where EyeGaze as a GF marks a certain type of Locative, the one that is interpreted as the endpoint in a telic predicate and not non-endpoint potential goal locatives (e.g., 'towards') ${ }^{6}$ We have further identified cases (as in (11)), where we find both EyeGaze on the endpoint locative and EyeGaze on the REACH head.

6. See also Rodriguez-Monroy (2021) for more details on this issue. 
These results, thus, provide support for the hypothesis in (5), and situate ASL as a doublemarking language in the typology of Nichols (1986), alongside languages like Euskera (in (4)) or Yup'ik (Jacobson 1995). While doing so, we show that there are no modality effects in this area, with the pattern of double-marking languages in both sign and spoken languages.

Whereas these results are consistent in our data, across participants and across prompts, it is not the goal of this work to identify frequency or preferences. The goal of this paper is to identify patterns allowed by the grammar of the language and any potential condition or restriction about them. Variation in agreement and Case-marking exists crosslinguistically: not all objects are marked accusative (Finnish for instance uses both accusative and partitive), ${ }^{7}$ nor do all subjects agree with their verb (in Arabic, for instance, postverbal subjects do not, whereas preverbal subjects do). ${ }^{8}$ It is our task in the future to further characterize the patterns we have observed here and see if any grammatical conditions are relevant.

As an example of Case variation, more relevant to our discussion here and to any potential issues of (cross-)modality effects, it is interesting to compare the pattern of EyeGaze as a GF marker of endpoint locatives with a pattern of Case alternation observed in German prepositional phrases. Certain prepositions in German have the choice of using either an Accusative Case or a Dative Case. The examples in (14) are an instance of this phenomenon: ${ }^{9}$
a. Das Boot trieb unter die Brücke.
The boat floats under the.AccF bridge
'The boat goes, floating, arriving [to the location] under the bridge.'

\section{b. Das Boot trieb unter der Brücke. \\ The boat floats under the.DATF bridge \\ 'The boat is [at the location] under the bridge, floating.'}

In (14a), the nominal constituent under the P-unter (die Brücke) appears in the (feminine) accusative form (realized in the determiner die). In (14b), on the other hand, the nominal constituent under the P-unter appears in the (feminine) dative form (again, realized in the determiner der). This contrast, however, is not 'free'; rather it carries a substantial change of meaning: in (14a) the PP unter die Brücke, with its accusative form, is to be interpreted as the endpoint of the motion denoted in the utterance (in (14a), thus, the verb is interpreted not just as a manner verb but as a displacement verb, carrying a trajectory); in (14b), on the contrary, the PP unter der Brücke is interpreted as a simple location (a locative of type ubi 'where-at' ${ }^{10}$ ). The use of the accusative in the endpoint locative PP in the German example of (14a) is, thus, parallel to the use of EyeGaze in endpoint locative in ASL in (6), both of them contrasting with non-endpoint locatives (the potential goal in the ASL (7), and the locativeubi in the German (14b). This crosslinguistic pattern of endpoint marking, including spoken and sign languages, show, again, that there is no modality effect in this area.

7. The distribution of partitive/accusative is quite complex in Finnish and it may be ultimately related to properties of the constituent and of the predicate (such as telicity).

8. Another well-known pattern of variation in agreement is the so-called ad-sensum agreement, as in a group of students was/were demanding some reforms, where the verb can agree with either group (in the singular) or students (in the plural).

9. Thanks to Roland Pfau (p.c.) for these German examples. A similar pattern can also be observed in Latin with prepositions that have flexible Case selection (e.g., Latin in, with Accusative and with Ablative).

10. Traditional grammar used this set of terms from Latin to identify the different types of location constituents: ubi 'where-at', quo 'where-to', unde 'where-from', qua 'where-through'. Later grammatical traditions inherited this system using the equivalents (relative/interrogative pronouns) in modern languages (e.g., 'wo', 'wohin', 'woher' in German; or 'dónde', 'adónde', 'de dónde' in Spanish). Unfortunately, the 'quo/ wohin/adónde’ does not distinguish endpoint locatives ('to/up-to') from potential target locatives ('towards'). 
In summary, the two areas analyzed in this paper: a. EyeGaze used as GF-marking on the constituent (Section 5.1), GF-marking on the head of the predicate (Section 5.2), and EyeGaze on both constituent and head underlying a double-marking language pattern; and b. EyeGaze as a contrasting GF-marker of endpoint locatives) reveal areas where the same morpho-syntactic patterns arise in both sign and spoken languages.

Therefore, we find that, as a double marking language and as a language contrasting endpoint locatives vs non-endpoint locatives, ASL patterns in alignment with the crosslinguistic variation observed elsewhere and, thus, no (cross-)modality effects are detected in these morpho-syntactic patterns.

\section{Acknowledgments}

Grateful thanks to: Participants; Robin Shay and members of the IELLab community; CLA / Purdue University for funding (Global Synergy Grant); Envision Center at Purdue University (for the video app).

\section{References}

Bahan, Benjamin. 1996. "Non-manual realization of agreement in American Sign Language." Doctoral dissertation, Boston University.

Benedicto, Elena. 2018. "Verbal Classifiers in Sign Languages... Agreement vs. AGREE?” In UMOP 40: The Leader of the Pack. A Festschrift in Honor of Peggy Speas, edited by Ivan Rodica, 1-18. Amherst: GLSA.

2019. Motion Predicates: Moving Along. A Tool for the Analysis of the Sub-Eventive Structure in Motion Predicates. https://doi.org/10.4231/R7PN93M4.

Benedicto, Elena, Chiara Branchini, and Lara Mantovan. 2015. "Decomposing the Internal Structure of Motion Predicates in Italian Sign Language (LIS).” In FEAST. Barcelona.

Benedicto, Elena, and Diane Brentari. 2004. "Where Did All the Arguments Go?: ArgumentChanging Properties of Classifiers in ASL." Natural Language \& Linguistic Theory 22 (4): 743-810.

Borer, Hagit. 2005. The Normal Course of Events. Oxford: Oxford University Press.

Brentari, Diane. 1993. "Establishing a Sonority Hierarchy in American Sign Language: The Use of Simultaneous Structure in Phonology.” Phonology 10 (2): 281-306. https: / / doi. org/10.1017/S0952675700000063.

Calderón Verde, Alicia, Donny Ansoccendsor@infomed.sld.cu Wilson Limonta, Ariel Hernández Hernández, and Elena E. Benedicto. 2018. "Path, Process and (a)Telicity in Space: Motion Predicates in LSCu, Sign Language of Cuba." FEAST. Formal and Experimental Advances in Sign language Theory 2. https:// doi.org/10.31009/FEAST.i2.01.

Emmorey, K., R. Thompson, and R. Colvin. 2008. "Eye Gaze during Comprehension of American Sign Language by Native and Beginning Signers." Journal of Deaf Studies and Deaf Education 14 (2): 237-243. https://doi.org/10.1093/deafed/enn037. 
Hosemann, Jana. 2013. "Eye Gaze and Verb Agreement in German Sign Language: A First Glance," edited by Annika Herrmann and Markus Steinbach, 73-90. Amsterdam: John Benjamins Publishing Company. https://doi.org/10.1075/bct.53.05hos.

Jacobson, Steven A. 1995. A Practical Grammar of the Central Alaskan Yup'ik Eskimo Language. Fairbanks: Alaska Native Language Center, University of Alaska Fairbanks.

Lourenço, Guilherme. 2018. "Verb Agreement in Brazilian Sign Language: Morphophonology, Syntax \& Semantics.” PhD diss., Universidade Federal de Minas Gerais.

Neidle, Carol, Judy Kegl, Dawn MacLaughlin, Benjamin Bahan, and Robert G. Lee. 2000. The Syntax of American Sign Language: Functional Categories and Hierarchical Structure. Cambridge: MIT Press.

Nichols, Johanna. 1986. "Head-Marking and Dependent-Marking Grammar." Language 62 (1): 56-119. https://doi.org/10.1353/lan.1986.0014.

Quadros, Ronice Müller de, and Josep Quer. 2011. “The Proper Characterization of Sign Language Agreement.” UFSC \& ICREA-University Pompeu Fabra.

Quer, Josep. 2010. "Signed Agreement: Putting the Arguments Together.” In Theoretical Issues in Sign Language Research 10. Purdue Univeristy.

Ramchand, Gillian. 2008. Verb Meaning and the Lexicon: A First Phase Syntax. Cambridge, MA: Cambridge University Press.

Rodriguez-Monroy, Paula. 2021. Asociación Del Rasgo No Manual de La Mirada Con La Telicidad En Predicados de Movimiento En La Lengua de Señas Americana.

Supalla, Ted. 1982. "Structure and acquisition of verbs of motion and location in American Sign Language.” Doctoral dissertation, University of California, San Diego.

Thompson, Robin, Karen Emmorey, and Robert Kluender. 2006. "The Relationship between Eye Gaze and Verb Agreement in American Sign Language: An Eye-Tracking Study.” Natural Language \& Linguistic Theory 24 (2): 571-604. https:// doi.org/10.1007/s11049-0051829-y. 